Roop Kaw, MD

Department of Hospital Medicine and Department

of Outcomes Research, Cleveland Clinic; Associate

Professor, Cleveland Clinic Lerner College of

Medicine of Case Western Reserve University,

Cleveland, $\mathrm{OH}$
Anthony G. Doufas, MD, PhD

Professor of Anesthesiology, Perioperative and

Pain Medicine, Stanford University School of

Medicine, Stanford, CA

\title{
Is regular oxygen supplementation safe for obese postoperative patients?
}

$\mathbf{P}$ OStOperative hypoxemia is common. A prospective blinded observational study ${ }^{1}$ found that $21 \%$ of noncardiac postoperative patients had a pulse oxygen saturation less than $90 \%$ for at least 10 minutes per hour in the first 48 hours after surgery, and 3\% had severe hypoxemia (pulse oxygen saturation < $80 \%$ for at least 30 minutes). Of note, most of the patients received supplemental oxygen for only a few hours after surgery, and standard observation by the nursing staff seriously underestimated the severity of hypoxemia.

Hypoxemia is more likely to occur in patients breathing room air as opposed to $35 \%$ oxygen. ${ }^{2}$ The number of cases detected and treated might be higher if patients were monitored by pulse oximetry, but correcting hypoxemia has not been shown to improve morbidity and mortality rates, cognitive function, or length of stay. ${ }^{3,4}$

Hence, oxygen supplementation has become the default clinical standard, as opposed to the more cumbersome and expensive option of continuous pulse oximetry after surgery.

\section{OBESITY, OBSTRUCTIVE SLEEP APNEA, AND CONTROL OF VENTILATION}

Obese patients are more likely to need oxygen after surgery, possibly because they are more likely to have obstructive sleep apnea, obesity hypoventilation syndrome, or physiologic restrictive lung disease. ${ }^{5}$ Patients with known obstructive sleep apnea are also more likely to receive supplemental oxygen postoperatively than to be resumed on continuous or bilevel positive airway pressure therapy.

The authors report no relevant financial relationships which, in the context of their contributions, could be perceived as a potential conflict of interest. doi:10.3949/ccjm.87a.19051
Complicating the picture, some patients with obstructive sleep apnea also have chronic obstructive pulmonary disease. Although supplemental oxygen may improve nocturnal oxygenation in patients with chronic obstructive pulmonary disease with only slight hypercapnia, hypercapnia may be more severe in patients who have both diseases, in which case giving oxygen may increase the duration of apnea episodes, leading to hypoventilation.

\section{Asleep vs awake}

Most patients with obstructive sleep apnea have enhanced chemoreflex sensitivity, and both obesity and metabolic syndrome have been shown to enhance ventilatory responses to hypoxia and hypercapnia-during wakefulness. ${ }^{6-8}$ On the other hand, obstructive sleep apnea in obese patients was associated with a blunted response to hypercapnia during sleep in a study by Yuan et al. ${ }^{9}$

This finding has been supported by evidence that opioid-induced ventilatory depression in postoperative bariatric patients is greater during sleep. ${ }^{10}$ Closer attention and ventilatory monitoring by the care staff in the immediate postoperative period, especially when patients are sedated or asleep, may help prevent the undesirable respiratory consequences of opioids.

\section{Supplemental oxygen in postoperative patients with obstructive sleep apnea}

Giving oxygen to patients with obstructive sleep apnea while they are asleep has been associated with variable outcomes, partly reflecting underlying differences in the mechanisms of ventilatory control. ${ }^{11}$

Liao et $\mathrm{al}^{12}$ performed a trial in postoperative obese patients with obstructive sleep apnea, randomizing them to receive either
Caution, but no evidence to support avoiding it altogether in obese patients 
supplemental oxygen at $3 \mathrm{~L} / \mathrm{min}$ or room air. The mean oxygen saturation was $95.2 \%$ with oxygen vs $91.4 \%$ with room air $(P<$ $.001)$, and the median apnea-hypopnea index was 8.0 vs $15.6(P=.016)$. The duration of apnea-hypopnea events was not increased, transcutaneous partial pressure of carbon dioxide increased significantly in only $11 \%$ of the patients on the first postoperative night, and no life-threatening events were reported. The total opioid requirement varied between 35 and $45 \mathrm{mg}$ morphine equivalents over a 72 hour period and did not differ between the 2 groups. Of note, only $3 \%$ of the patients had a diagnosis of chronic obstructive pulmonary disease, and patients with presumed obesity hypoventilation syndrome (based on a serum bicarbonate level $>30 \mathrm{mmol} / \mathrm{L}$ ) were excluded from the study.

\section{Different phenotypes of obstructive sleep apnea?}

Differences in response to supplemental oxygen in patients with obstructive sleep apnea have been attributed to differences in "loop gain," an engineering term that describes the gain of the negative feedback loop that regulates ventilation. ${ }^{13}$ If loop gain is high, ventilatory control is relatively unstable, and vice versa. Giving oxygen lowers a high loop gain, hence leading to moderation (or elimination) of an excessive postobstruction hyperventilatory response that may have precipitated further hypocapnia-related cycles of airway obstruction.

Wellman et $\mathrm{al}^{13}$ gave supplemental oxygen overnight to 12 patients with obstructive sleep apnea (not postoperative patients), of whom 6 had high loop gain and 6 had low. Oxygen lowered the apnea-hypopnea index in those with high loop gain but not in those with low loop gain.

At present, however, the extent to which certain phenotypes of obstructive sleep apnea influence the risk of adverse postoperative outcomes in obese patients remains unknown.

\section{OPIOIDS AND OXYGEN}

Postoperative patients experience a state of relative hypoventilation due to the residual effects of anesthesia and to opioid analgesics. Depending on the dose and route of administration, patient-related characteristics, the monitoring method, and the definition used, the incidence of postoperative opioid-induced ventilatory depression ranges from less than $1 \%$ to up to $40 \%$. $114-16$

In a series of 92 cases of severe opioidinduced ventilatory depression associated with significant morbidity and mortality that were identified through insurance malpractice claims, ${ }^{17}$ only a third of the patients were monitored with pulse oximetry, and only $15 \%$ were receiving supplemental oxygen; $42 \%$ of these events occurred within 2 hours of the last nursing check, and only a quarter of the patients either had a diagnosis of obstructive sleep apnea or were at high risk of it (with a STOP-BANG score $\geq 3$ ). This evidence suggests that during hypoxemia, patients may benefit from closer monitoring, supplemental oxygen, or both to prevent hypoxic insults from escalating to more serious morbidity.

Similarly, a randomized trial ${ }^{18}$ comparing supplemental oxygen at a regular flow rate (2-4 L/min to maintain pulse oxygen saturation $\geq 93 \%)$ vs a high flow rate $(45 \mathrm{~L} / \mathrm{min})$ in postcardiac surgery patients did not show any advantage in postoperative oxygenation, but decreased the need for escalation of respiratory support.

Intermittent pulse oximetry has been shown to substantially underestimate ventilatory depression compared with continuous oximetry and capnography monitoring. ${ }^{1,19}$

Recently, investigators in an international prospective trial ${ }^{16}$ (Prediction of Opioidinduced Respiratory Depression in Patients Monitored by Capnography; PRODIGY) developed a risk tool to predict opioid-induced respiratory depression. It is derived from data from 1,335 hospitalized patients on general medical-surgical floors who were monitored by continuous capnography and oximetry, of whom $614(46 \%)$ had 1 or more episodes of respiratory depression. Points are awarded for age, male sex, no prior use of opioids, sleep disordered breathing, and chronic heart failure. Patients with a high PRODIGY score $(\geq 15$ of a possible 39) were more likely to develop respiratory depression than those with a score less than 8 (odds ratio 6.07, 95\% confidence interval 4.44-8.30). The area under the receiver-operating curve was 0.74 . Patients with respiratory depression were 2.5 times more 
likely to need rescue action (including rapidresponse-team activation) and 1.4 times more likely to need prolonged hospitalization.

Pharmacologic models need to be developed and validated for opioids in morbidly obese patients to link drug dose and effect and to interrogate the physiology behind the differential sensitivity of these patients for opioid-induced analgesia, sedation, and ventilatory depression.

\section{DOES SUPPLEMENTAL OXYGEN MASK HYPOVENTILATION?}

In spite of evidence that supplemental oxygen improves oxygenation in postoperative patients receiving opioids by patient-controlled devices, concern has been expressed that it could hamper our ability to promptly detect opioid-induced ventilatory depression and thus prevent morbid outcomes. This concern is based on experimental ${ }^{20}$ and clinical ${ }^{19,21,22}$ evidence that, in contrast to room air, oxygen supplementation may delay oxygen desaturation associated with ventilatory depression and associated hypercapnia.

Thus, some have suggested giving oxygen, but no more than 30\% (which would increase arterial oxygen tension from $30 \mathrm{~mm} \mathrm{Hg}$ to 94 $\mathrm{mm} \mathrm{Hg}$, with a carbon dioxide tension of 98 $\mathrm{mm} \mathrm{Hg}$ ), when a patient who is breathing room air (which contains $21 \%$ oxygen) becomes hypoxemic due to hypoventilation..$^{23}$

On the other hand, Taenzer et $\mathrm{al}^{24}$ demonstrated that, compared with room air, supplemental oxygen at 1 to $6 \mathrm{~L} / \mathrm{min}$ neither influenced the magnitude or duration of desaturation events nor impaired the effectiveness of pulse oxygen saturation monitoring in detecting those events in postoperative patients.

The PRODIGY trial ${ }^{16}$ reported a higher overall incidence of opioid-induced ventilatory depression $(46 \%)$ than other studies, possibly because the patients underwent combined continuous capnography and oximetry monitoring, which detected more apneic and hypoventilation episodes. Notably, only $8 \%$ of the patients with episodes of opioid-induced ventilatory depression experienced hypoxemia. This low incidence was attributed to use of supplemental oxygen in most of the patients.
Current evidence does not agree as to the best monitoring method for promptly detecting a potentially serious respiratory event. An array of monitors may be the answer, to the extent that information they produce is both predictive and congruent between the instruments. Taenzer et al, ${ }^{25,26}$ in an opportunity cost-based analysis modeled only on reduction of intensive care unit transfers and days spent in intensive care, reported that although universal continuous monitoring using a patient surveillance system would be cost-effective on certain units like the thoracovascular unit, it would be neutral or even more costly on medical units.

\section{OBESITY HYPOVENTILATION SYNDROME AND $100 \%$ OXYGEN}

A double-blind, randomized, placebo-controlled crossover study ${ }^{27}$ concluded by advising extreme caution in giving 100\% oxygen to patients with suspected but untreated obesity hypoventilation syndrome and reported a significant decrease in minute ventilation with consequent worsening of hypercapnia, as measured by transcutaneous carbon dioxide tension. The carbon dioxide tension rose by $5 \mathrm{~mm} \mathrm{Hg}$ after 20 minutes, and a few patients showed a higher rate of rise, needing withdrawal from the study. However, there are no observations beyond 20 minutes to determine if carbon dioxide would continue to rise or respiratory acidosis ensue.

Up to one-third of morbidly obese patients may have hypercapnia from presumed obesity hypoventilation syndrome, which is often unrecognized. ${ }^{27}$ The lower the pulse oxygen saturation in obese patients, the more likely they are to receive a high fraction of inspired oxygen, potentially leading to a larger increase in hypercapnia. Also, up to $40 \%$ of patients with obesity hypoventilation syndrome may need additional oxygen in a nonsurgical environment, despite being adequately treated with positive airway pressure. ${ }^{28}$ Interestingly, in patients who have obesity hypoventilation syndrome with hypoxia during sleep despite adequate long-term noninvasive positive-pressure ventilation therapy, supplemental oxygen therapy was found to be the only independent predictor of death. ${ }^{29}$

No studies to date have reported on the effect of supplemental oxygen in patients with
The PRODIGY risk tool predicts opioid-induced respiratory depression 
obesity hypoventilation syndrome while they are treated with intravenous opioids in the postoperative period, nor are there any data reporting the effect of high oxygen concentration or flow $(>3 \mathrm{~L} / \mathrm{min}$ ) in postoperative patients with obstructive sleep apnea receiving intravenous opioids for pain. Recently, however, it has been shown that patients with obstructive sleep apnea associated with hypercapnia had worse postoperative outcomes than those with obstructive sleep apnea alone, regardless of the severity of the sleep apnea or the body mass index. ${ }^{30}$

\section{CONCLUSIONS}

We advise caution in giving high-flow supple-

\section{REFERENCES}

1. Sun Z, Sessler DI, Dalton JE, et al. Postoperative hypoxemia is common and persistent: a prospective blinded observational study. Anesth Analg 2015; 121(3):709-715. doi:10.1213/ANE.0000000000000836

2. Canet J, Ricos M, Vidal F. Early postoperative arterial oxygen desaturation. Determining factors and response to oxygen therapy. Anesth Analg 1989; 69(2):207-212. pmid:2764289

3. Pedersen T, Nicholson A, Hovhannisyan K, Møller AM, Smith AF, Lewis SR. Pulse oximetry for perioperative monitoring. Cochrane Database Syst Rev 2014; 2014(3):CD002013. doi:10.1002/14651858.CD002013.pub3

Up to one-third of morbidly obese patients may have hypercapnia from presumed obesity hypoventilation syndrome
4. Moller JT, Johannessen NW, Espersen K, et al. Randomized evaluation of pulse oximetry in 20,802 patients: II. Perioperative events and postoperative complications. Anesthesiology 1993; 78(3):445-453. doi:10.1097/00000542-199303000-00007

5. American Society of Anesthesiologists Task Force on Perioperative Management of patients with obstructive sleep apnea. Practice guidelines for the perioperative management of patients with obstructive sleep apnea: an updated report by the American Society of Anesthesiologists Task Force on Perioperative Management of patients with obstructive sleep apnea. Anesthesiology 2014; 120(2):268-286. doi:10.1097/ALN.0000000000000053

6. Narkiewicz K, Kato M, Pesek CA, Somers VK. Human obesity is characterized by a selective potentiation of central chemoreflex sensitivity. Hypertension 1999; 33(5):1153-1158. doi:10.1161/01.hyp.33.5.1153

7. Narkiewicz K, van de Borne PJ, Pesek CA, Dyken ME, Montano N, Somers VK. Selective potentiation of peripheral chemoreflex sensitivity in obstructive sleep apnea. Circulation 1999; 99(9):1183-1189. doi:10.1161/01.cir.99.9.1183

8. Trombetta IC, Maki-Nunes C, Toschi-Dias E, et al. Obstructive sleep apnea is associated with increased chemoreflex sensitivity in patients with metabolic syndrome. Sleep 2013; 36(1):41-49. doi:10.5665/sleep.2298

9. Yuan H, Pinto SJ, Huang J, et al. Ventilatory responses to hypercapnia during wakefulness and sleep in obese adolescents with and without obstructive sleep apnea syndrome. Sleep 2012; 35(9):1257-1267. doi:10.5665/sleep.2082

10. Zaremba S, Shin CH, Hutter MM, et al. Continuous positive airway pressure mitigates opioid-induced worsening of sleep-disordered breathing early after bariatric mental oxygen to obese postoperative patients who have known or suspected obstructive sleep apnea or obesity hypoventilation syndrome, especially when they are sedated, asleep, or receiving intravenous opioids. The concern is that giving high concentration oxygen could mask hypoventilation and hypoxemia, lead to greater hypercapnia, and delay needed treatment.

That said, there is no evidence to date to support withholding supplemental oxygen altogether in obese patients, even those with obesity hypoventilation syndrome, although evidence regarding the optimal fraction of inspired oxygen or target oxygen saturation in these patients is currently lacking.

surgery. Anesthesiology 2016; 125(1):92-104 doi:10.1097/ALN.0000000000001160

11. Doufas AG. Obstructive sleep apnea, pain, and opioid analgesia in the postoperative patient. Curr Anesthesiol Rep 2014; 4:1-9. doi:10.1007/s40140-013-0047-0

12. Liao $\mathbf{P}$, Wong J, Singh $\mathbf{M}$, et al. Postoperative oxygen therapy in patients with OSA: a randomized controlled trial. Chest 2017; 151(3):597-611. doi:10.1016/j.chest.2016.12.005

13. Wellman A, Malhotra A, Jordan AS, Stevenson KE, Gautam S, White DP. Effect of oxygen in obstructive sleep apnea: role of loop gain. Respir Physiol Neurobiol 2008; 162(2):144-151. doi:10.1016/j.resp.2008.05.019

14. Cashman JN, Dolin SJ. Respiratory and haemodynamic effects of acute postoperative pain management: evidence from published data. Br J Anaesth 2004; 93(2):212-223. doi:10.1093/bja/aeh180

15. Belcher AW, Khanna AK, Leung S, et al. Long-acting patient-controlled opioids are not associated with more postoperative hypoxemia than short-acting patientcontrolled opioids after noncardiac surgery: a cohort analysis. Anesth Analg 2016; 123(6):1471-1479. doi:10.1213/ANE.0000000000001534

16. Khanna AK, Bergese SD, Jungquist CR, et al. Prediction of opioid-induced respiratory depression on inpatient wards using continuous capnography and oximetry: an international prospective, observational trial. Anesth Analg 2020; April 16. doi:10.1213/ANE.0000000000004788

17. Lee LA, Caplan RA, Stephens LS, et al. Postoperative opioid-induced respiratory depression: a closed claims analysis. Anesthesiology 2015; 122(3):659-665. doi:10.1097/ALN.0000000000000564

18. Parke R, McGuinness S, Dixon R, Jull A. Open-label, phase II study of routine high-flow nasal oxygen therapy in cardiac surgical patients. Br J Anaesth 2013; 111(6):925-931. doi:10.1093/bja/aet262

19. Lam T, Nagappa M, Wong J, Singh M, Wong D, Chung F. Continuous pulse oximetry and capnography monitoring for postoperative respiratory depression and adverse events: a systematic review and meta-analysis. Anesth Analg 2017; 125(6):2019-2029. doi:10.1213/ANE.0000000000002557

20. Niesters M, Mahajan RP, Aarts L, Dahan A. High-inspired oxygen concentration further impairs opioid-induced re spiratory depression. Br J Anaesth 2013; 110(5):837-841. doi:10.1093/bja/aes494

21. Kopka A, Wallace E, Reilly G, Binning A. Observational 
study of perioperative $\mathrm{PtcCO} 2$ and $\mathrm{SpO} 2$ in non-ventilated patients receiving epidural infusion or patient-controlled analgesia using a single earlobe monitor (TOSCA). Br J Anaesth 2007; 99(4):567-571.

doi:10.1093/bja/aem206

22. Fu ES, Downs JB, Schweiger JW, Miguel RV, Smith RA. Supplemental oxygen impairs detection of hypoventilation by pulse oximetry. Chest 2004; 126(5):1552-1558. doi:10.1378/chest.126.5.1552

23. Lumb AB. Oxygen. In: Lumb AB, ed. Nunn's Applied Respiratory Physiology. 6th ed. Elsevier; 2005:166-200.

24. Taenzer AH, Perreard IM, MacKenzie T, McGrath SP. Characteristics of desaturation and respiratory rate in postoperative patients breathing room air versus supplemental oxygen: are they different? Anesth Analg 2018; 126(3):826-832. doi:10.1213/ANE.0000000000002765

25. Taenzer AH, Pyke JB, McGrath SP, Blike GT. Impact of pulse oximetry surveillance on rescue events and intensive care unit transfers: a before-and-after concurrence study. Anesthesiology 2010; 112(2):282-287.

doi:10.1097/ALN.0b013e3181ca7a9b

26. Taenzer AH, Blike GT; APSF Newsletter. Postoperative monitoring - the Dartmouth experience. https://www. apsf.org/article/postoperative-monitoring-the-dartmouth-experience/. Accessed November 5, 2020.

27. Wijesinghe $\mathbf{M}$, Williams $\mathbf{M}$, Perrin $\mathrm{K}$, Weatherall $\mathbf{M}$, Beasley R. The effect of supplemental oxygen on hypercapnia in subjects with obesity-associated hypoventilation: a randomized, crossover, clinical study. Chest 2011; 139(5):1018-1024. doi:10.1378/chest.10-1280

28. Banerjee D, Yee BJ, Piper AJ, Zwillich CW, Grunstein RR. Obesity hypoventilation syndrome: hypoxemia during continuous positive airway pressure. Chest 2007; 131(6):1678-1684. doi:10.1378/chest.06-2447

29. Priou P, Hamel JF, Person C, et al. Long-term outcome of noninvasive positive pressure ventilation for obesity hypoventilation syndrome. Chest 2010; 138(1):84-90. doi:10.1378/chest.09-2472

30. Kaw R, Bhateja P, Paz Y Mar H, et al. Postoperative complications in patients with unrecognized obesity hypoventilation syndrome undergoing elective noncardiac surgery. Chest 2016; 149(1):84-91. doi:10.1378/chest.14-3216

Address: Roop Kaw, MD, Department of Hospital Medicine, M2 Annex, Cleveland Clinic, 9500 Euclid Avenue, Cleveland, $\mathrm{OH}$ 44195; kawr@ccf.org 